Honam Mathematical J. 35 (2013), No. 2, pp. 109-117

http://dx.doi.org/10.5831/HMJ.2013.35.2.109

\title{
FIXED POINTS OF CONVERSE COMMUTING MAPPINGS USING AN IMPLICIT RELATION
}

\author{
Sunny Chauhan, M. Alamgir Khan and \\ Wutiphol Sintunavarat
}

\begin{abstract}
In the present paper, we utilize the notion of converse commuting mappings due to Lü [On common fixed points for converse commuting self-maps on a metric spaces, Acta. Anal. Funct. Appl. 4(3) (2002), 226-228] and prove a common fixed point theorem in Menger space using an implicit relation. We also give an illustrative example to support our main result.
\end{abstract}

\section{Introduction}

In 1986, Jungck [9] introduced the notion of compatible mappings in metric space. Most of the common fixed point theorems for contraction mappings invariably require a compatibility condition besides assuming continuity of at least one of the mappings. Later on, Jungck and Rhoades [10] studied the notion of weakly compatible mappings and utilized it as a tool to improve commutativity conditions in common fixed point theorems. Many mathematicians proved several fixed point results in Menger spaces (see $[2,3,4,6,7,8,16,18,19,20,25]$ ). In 2002, Lü [13] presented the concept of the converse commuting mappings, as a reverse process of weakly compatible mappings and proved common fixed point theorems for single-valued mappings in metric spaces (also see [14]). Recently, Pathak and Verma [21, 22] and Chugh et al. [5] proved some fixed point theorems for converse commuting mappings.

In 1998, Popa and Turkoğlu [24] proved some fixed points theorem for hybrid mappings by using an implicit relation. Popa used the family of implicit real functions and proved common fixed point theorems (also see, [23]).

Received September 8, 2012. Accepted April 25, 2013.

2010 Mathematics Subject Classification. Primary 47H10, Secondary 54H25.

Key words and phrases. $t$-norm, Menger space, weakly compatible mappings, converse commuting mappings, implicit relation, fixed point. 
The aim of this paper is to prove a common fixed point theorem for two pairs of converse commuting mappings in Menger space using an implicit relation. An illustrative example to highlight the realized improvements is furnished.

\section{Preliminaries}

Definition 2.1. ([26]) A mapping $\triangle:[0,1] \times[0,1] \rightarrow[0,1]$ is said to be t-norm if $\triangle$ is satisfying the following conditions:

1. $\triangle$ is commutative and associative;

2. $\triangle(a, 1)=a$ for all $a \in[0,1]$;

3. $\triangle(a, b) \leq \triangle(c, d)$ whenever $a \leq c$ and $b \leq d$ for all $a, b, c, d \in[0,1]$.

Examples of t-norms are $\triangle(a, b)=\min \{a, b\}, \triangle(a, b)=a b$ and $\triangle(a, b)=\max \{a+b-1,0\}$.

Definition 2.2. ([26]) A mapping $F: \mathbb{R} \rightarrow \mathbb{R}^{+}$is called a distribution function if it is non-decreasing and left continuous with $\inf \{F(t): t \in$ $\mathbb{R}\}=0$ and $\sup \{F(t): t \in \mathbb{R}\}=1$.

We shall denote by $\Im$ the set of all distribution functions defined on $(-\infty, \infty)$ while $H(t)$ will always denote the specific distribution function defined by

$$
H(t)= \begin{cases}0, & \text { if } t \leq 0 ; \\ 1, & \text { if } t>0 .\end{cases}
$$

If $X$ is a non-empty set, $\mathcal{F}: X \times X \rightarrow \Im$ is called a probabilistic distance on $X$ and the value of $\mathcal{F}$ at $(x, y) \in X \times X$ is represented by $F_{x, y}$.

Definition 2.3. ([15]) A probabilistic metric space is an ordered pair $(X, \mathcal{F})$, where $X$ is a non-empty set of elements and $\mathcal{F}$ is a probabilistic distance satisfying the following conditions: for all $x, y, z \in X$ and $t, s>$ 0 ,

1. $F_{x, y}(t)=H(t)$ for all $t>0$ if and only $x=y$;

2. $F_{x, y}(t)=F_{y, x}(t)$;

3. if $F_{x, y}(t)=1$ and $F_{y, z}(s)=1$, then $F_{x, z}(t+s)=1$.

Every metric space $(X, d)$ can always be realized as a probabilistic metric space by considering $\mathcal{F}: X \times X \rightarrow \Im$ defined by $F_{x, y}(t)=$ $H(t-d(x, y))$ for all $x, y \in X$. So probabilistic metric spaces offer a wider framework than that of metric spaces and are better suited to cover even wider statistical situations. 
Definition 2.4. ([26]) A Menger space $(X, \mathcal{F}, \triangle)$ is a triplet where $(X, \mathcal{F})$ is a probabilistic metric space and $\triangle$ is a t-norm satisfying the following condition:

$$
F_{x, y}(t+s) \geq \triangle\left(F_{x, z}(t), F_{z, y}(s)\right)
$$

for all $x, y, z \in X$ and $t, s>0$.

Definition 2.5. ([10]) Self mappings $A$ and $S$ of a non-empty set $X$ are said to be weakly compatible (or coincidentally commuting) if they commute at their coincidence points, that is, if $A x=S x$ for some $x \in X$, then $A S x=S A x$.

Definition 2.6. ([13]) Self mappings $A$ and $S$ of a non-empty set $X$ are called conversely commuting if, for all $x \in X, A S x=S A x$ implies $A x=S x$.

Definition 2.7. ([13]) Let $A$ and $S$ be self mappings of a non-empty set $X$. A point $x \in X$ is called commuting point of $A$ and $S$ if $A S x=$ $S A x$.

Lemma 2.8. ([17]) Let $(X, \mathcal{F}, \triangle)$ be a Menger space. If there exists a constant $k \in(0,1)$ such that

$$
F_{x, y}(k t) \geq F_{x, y}(t)
$$

for all $t>0$ with fixed $x, y \in X$ then $x=y$.

\section{Implicit Relation}

Many authors proved a number of common fixed point theorems using the notion of implicit relation on different spaces (see [1], [11], [12], [23], [24], [27]).

Let $I=[0,1], \triangle$ be a continuous t-norm and $\varphi: I^{6} \rightarrow \mathbb{R}$ be a continuous function. Now, we consider the following conditions:

$(\varphi-1) \varphi$ is non-increasing in the fifth and sixth variables,

$(\varphi-2)$ If, for some constant $k \in(0,1)$, we have

$$
\text { or } \quad \begin{aligned}
& \left(\varphi_{a}\right) \quad \varphi\left(u(k t), v(t), v(t), u(t), 1, \triangle\left(u\left(\frac{t}{2}\right), v\left(\frac{t}{2}\right)\right)\right) \geq 1 \\
& \quad\left(\varphi_{b}\right) \quad \varphi\left(u(k t), v(t), u(t), v(t), \triangle\left(u\left(\frac{t}{2}\right), v\left(\frac{t}{2}\right)\right), 1\right) \geq 1
\end{aligned}
$$

for any fixed $t>0$ and any non-decreasing functions $u, v:(0, \infty) \rightarrow$ $I$ with $0<u(t), v(t) \leq 1$, then there exists $h \in(0,1)$ with $u(h t) \geq$ $\triangle(v(t), u(t))$.

$(\varphi-3)$ If, for some constant $k \in(0,1)$, we have

$$
\varphi(u(k t), u(t), 1,1, u(t), u(t)) \geq 1
$$


for any fixed $t>0$ and any non-decreasing function $u:(0, \infty) \rightarrow I$, then $u(k t) \geq u(t)$.

Now, let $\Phi$ be the set of all real continuous functions $\varphi: I^{6} \rightarrow \mathbb{R}$ satisfying the conditions $(\varphi-1) \sim(\varphi-3)$.

Example 3.1. ([1]) Let $\varphi\left(u_{1}, \ldots, u_{6}\right)=\frac{u_{1}}{\min \left\{u_{2}, \ldots, u_{6}\right\}}$ and $\triangle(a, b)=$ $\min \{a, b\}$.

Let $t>0,0<u(t), v(t) \leq 1, k \in\left(0, \frac{1}{2}\right)$, where $u, v:[0, \infty) \rightarrow I$ are non-decreasing functions. Now, suppose that

$$
\varphi\left(u(k t), v(t), v(t), u(t), 1, \triangle\left(u\left(\frac{t}{2}\right), v\left(\frac{t}{2}\right)\right)\right) \geq 1,
$$

i.e.,

$$
\begin{aligned}
\varphi\left(u(k t), v(t), v(t), u(t), 1, \triangle\left(u\left(\frac{t}{2}\right), v\left(\frac{t}{2}\right)\right)\right) & =\frac{u(k t)}{\min \left\{v(t), u(t), 1, \triangle\left(u\left(\frac{t}{2}\right), v\left(\frac{t}{2}\right)\right)\right\}} \\
& =\frac{u(k t)}{\min \left\{v\left(\frac{t}{2}\right), u\left(\frac{t}{2}\right)\right\}} \geq 1 .
\end{aligned}
$$

Thus $u(h t) \geq \triangle(v(t), u(t))$ if $h=2 k \in(0,1)$. A similar argument works if $\left(\varphi_{b}\right)$ is assumed. Finally, suppose that $t>0$ is fixed, $u$ : $(0, \infty) \rightarrow I$ is a non-decreasing function and

$$
\varphi(u(k t), u(t), 1,1, u(t), u(t))=\frac{u(k t)}{u(t)} \geq 1
$$

for some $k \in(0,1)$. Then we have $u(k t) \geq u(t)$ and thus $\varphi \in \Phi$.

Example 3.2. ([1]) Let $\varphi\left(u_{1}, \ldots, u_{6}\right)=\frac{u_{1} \max \left\{u_{2}, u_{3}, u_{4}\right\}}{\min \left\{u_{5}, u_{6}\right\}}$ and $\triangle$ be a continuous t-norm.

Let $t>0,0<u(t), v(t) \leq 1, k \in\left(0, \frac{1}{2}\right)$, where $u, v:[0, \infty) \rightarrow I$ are non-decreasing functions. Now, suppose that

$$
\varphi\left(u(k t), v(t), v(t), u(t), 1, \triangle\left(u\left(\frac{t}{2}\right), v\left(\frac{t}{2}\right)\right)\right) \geq 1,
$$

i.e.,

$\varphi\left(u(k t), v(t), v(t), u(t), 1, \triangle\left(u\left(\frac{t}{2}\right), v\left(\frac{t}{2}\right)\right)\right)=\frac{u(k t) \max \{v(t), u(t)\}}{\triangle\left(u\left(\frac{t}{2}\right), v\left(\frac{t}{2}\right)\right)} \geq 1$.

Thus $u(h t) \geq \triangle(v(t), u(t))$ if $h=2 k \in(0,1)$. A similar argument works if $\left(\varphi_{b}\right)$ is assumed. Finally, suppose that $t>0$ is fixed, $u$ : $(0, \infty) \rightarrow I$ is a non-decreasing function and

$$
\varphi(u(k t), u(t), 1,1, u(t), u(t))=\frac{u(k t)}{u(t)} \geq 1
$$

for some $k \in(0,1)$. Then we have $u(k t) \geq u(t)$ and thus $\varphi \in \Phi$. 
Example 3.3. ([1]) Let $\varphi\left(u_{1}, \ldots, u_{6}\right)=\frac{\left(u_{1}\right)^{3}}{\triangle\left(u_{2}, \triangle\left(u_{3}, u_{4}\right)\right) \max \left\{u_{5}, u_{6}\right\}}$ and $\triangle(a, b)=a b$.

Let $t>0,0<u(t), v(t) \leq 1, k \in(0,1)$, where $u, v:[0, \infty) \rightarrow I$ are non-decreasing functions. Now, suppose that

$$
\varphi\left(u(k t), v(t), v(t), u(t), 1, \triangle\left(u\left(\frac{t}{2}\right), v\left(\frac{t}{2}\right)\right)\right) \geq 1
$$

i.e.,

$$
\varphi\left(u(k t), v(t), v(t), u(t), 1, \triangle\left(u\left(\frac{t}{2}\right), v\left(\frac{t}{2}\right)\right)\right)=\frac{(u(k t))^{3}}{(v(t))^{2} u(t)} \geq 1 .
$$

Thus we have

$$
u(k t)=u(h t) \geq\left((v(t))^{\frac{2}{3}}(u(t))^{\frac{1}{3}}\right) \geq v(t) u(t)=\triangle(v(t), u(t)),
$$

if $h=k \in(0,1)$. A similar argument works if $\left(\varphi_{b}\right)$ is assumed. Finally, suppose that $t>0$ is fixed, $u:(0, \infty) \rightarrow I$ is a non-decreasing function and

$$
\varphi(u(k t), u(t), 1,1, u(t), u(t))=\frac{(u(k t))^{3}}{(u(t))^{2}} \geq 1
$$

for some $k \in(0,1)$. Then we have $u(k t) \geq u(t)$ and thus $\varphi \in \Phi$.

\section{Main Result}

Theorem 4.1. Let $A, B, S$ and $T$ be four self mappings on a Menger space $(X, \mathcal{F}, \triangle)$, where $\triangle$ is a continuous t-norm such that the pairs $(A, S)$ and $(B, T)$ are each conversely commuting satisfying

(1) $\varphi\left(F_{A x, B y}(k t), F_{S x, T y}(t), F_{A x, S x}(t), F_{B y, T y}(t), F_{A x, T y}(t), F_{B y, S x}(t)\right) \geq 1$ for all $x, y \in X, t>0$, where $k \in(0,1)$ and $\varphi \in \Phi$. If $A$ and $S$ have a commuting point and $B$ and $T$ have a commuting point, then $A, B, S$ and $T$ have a unique common fixed point in $X$.

Proof. Let $u$ be the commuting point of $A$ and $S$ and $v$ be the commuting point of $B$ and $T$. Since $A$ and $S$ are converse commuting we have $A S u=S A u \Rightarrow A u=S u$ and $B T v=T B v \Rightarrow B v=T v$. Hence $A A u=A S u=S A u=S S u$ and $B B v=B T v=T B v=T T v$. First we assert that $A u=B v$. To accomplish this, using (1) with $x=u, y=v$, we have

$\varphi\left(F_{A u, B v}(k t), F_{S u, T v}(t), F_{A u, S u}(t), F_{B v, T v}(t), F_{A u, T v}(t), F_{B v, S u}(t)\right) \geq 1$, or, equivalently,

$$
\varphi\left(F_{A u, B v}(k t), F_{A u, B v}(t), 1,1, F_{A u, B v}(t), F_{B v, A u}(t)\right) \geq 1 .
$$


Thus, from $(\varphi-3)$, we get

$$
F_{A u, B v}(k t) \geq F_{A u, B v}(t) .
$$

On employing Lemma 2.8, we obtain $A u=B v$. Therefore, $A u=$ $S u=B v=T v$. Now, we show that $A u$ is a fixed point of $A$. In order to establish this, using (1) with $x=A u, y=v$, we have

$\varphi\left(F_{A A u, B v}(k t), F_{S A u, T v}(t), F_{A A u, S A u}(t), F_{B v, T v}(t), F_{A A u, T v}(t), F_{B v, S A u}(t)\right) \geq 1$, and so

$$
\varphi\left(F_{A A u, A u}(k t), F_{A A u, A u}(t), 1,1, F_{A A u, A u}(t), F_{A u, A A u}(t)\right) \geq 1 .
$$

Thus, from $(\varphi-3)$, we get

$$
F_{A A u, A u}(k t) \geq F_{A A u, A u}(t) .
$$

Appealing to Lemma 2.8, we obtain $A A u=A u$. Similarly we have $B v=B B v$. Since $A u=B v$, we have $A u=B v=B B v=B A u$ which shows that $A u$ is a fixed point of mapping $B$.

On the other hand, $A u=B v=B B v=T B v=T A u$ and $A u=$ $A A u=A S u=S A u$. Hence $A u$ is a common fixed point of $A, B, S$ and $T$.

For the uniqueness of common fixed point, we use (1) with $x=u$ and $y=\widehat{u}$ such that $\widehat{u}$ is an another common fixed point of $A, B, S$ and $T$. Now we have

$\varphi\left(F_{A A u, B \widehat{u}}(k t), F_{S A u, T \widehat{u}}(t), F_{A A u, S A u}(t), F_{B \widehat{u}, T \widehat{u}}(t), F_{A A u, T \widehat{u}}(t), F_{B \widehat{u}, S A u}(t)\right) \geq 1$, and so

$$
\varphi\left(F_{A A u, A \widehat{u}}(k t), F_{A A u, B \widehat{u}}(t), 1,1, F_{A A u, A u}(t), F_{A u, A A u}(t)\right) \geq 1 .
$$

Again, from $(\varphi-3)$, we get

$$
F_{A A u, A \widehat{u}}(k t) \geq F_{A A u, A \widehat{u}}(t) .
$$

By Lemma 2.8, we get $A A u=A \widehat{u}$. Therefore, $u=A u=A A u=$ $A \widehat{u}=\widehat{u}$. Thus $u$ is a unique common fixed point of $A, B, S$ and $T$.

Now, we give an example which illustrates Theorem 4.1.

Example 4.2. Let $X=[1, \infty)$ with the metric $d$ defined by $d(x, y)=$ $|x-y|$ and for each $t \in[0,1]$, define

$$
F_{x, y}(t)= \begin{cases}\frac{t}{t+|x-y|}, & \text { if } t>0 ; \\ 0, & \text { if } t=0,\end{cases}
$$

for all $x, y \in X$. Clearly $(X, \mathcal{F}, \triangle)$ be a Menger space, where $\triangle$ is a continuous t-norm. Let $\varphi: I^{6} \rightarrow \mathbb{R}$ be defined as in Example 3.1 and define the self mappings $A, B, S$ and $T$ by 


$$
\begin{gathered}
A(x)=\left\{\begin{array}{ll}
2 x-1, & \text { if } x<2 ; \\
1, & \text { if } x \geq 2 .
\end{array} \quad S(x)= \begin{cases}x^{2}, & \text { if } x<2 ; \\
x+3, & \text { if } x \geq 2 .\end{cases} \right. \\
B(x)=\left\{\begin{array}{ll}
2 x-1, & \text { if } x<2 ; \\
2, & \text { if } x \geq 2 .
\end{array} \quad T(x)= \begin{cases}3 x^{2}-2, & \text { if } x<2 ; \\
x^{2}+1, & \text { if } x \geq 2 .\end{cases} \right.
\end{gathered}
$$

Hence the pairs $(A, S)$ and $(B, T)$ are converse commuting and 1 is a unique common fixed point of $A, B, S$ and $T$.

On taking $A=B$ and $S=T$ in Theorem 4.1, we get the following natural result.

Corollary 4.3. Let $A$ and $S$ be two self mappings on a Menger space $(X, \mathcal{F}, \triangle)$, where $\triangle$ is a continuous t-norm such that the pair $(A, S)$ is conversely commuting satisfying

(2) $\varphi\left(F_{A x, A y}(k t), F_{S x, S y}(t), F_{A x, S x}(t), F_{A y, S y}(t), F_{A x, S y}(t), F_{A y, S x}(t)\right) \geq 1$

for all $x, y \in X, t>0$, where $k \in(0,1)$ and $\varphi \in \Phi$. If $A$ and $S$ have a commuting point, then $A$ and $S$ have a unique common fixed point in $X$.

\section{Acknowledgments}

The authors would like to express their sincere thanks to Professor Xin-qi Hu for his paper [14]. Special thanks are also due to the reviewers, who have made a number of valuable comments and suggestions which have improved the manuscript greatly.

\section{References}

[1] I. Altun and D. Turkoğlu, Some fixed point theorems on fuzzy metric spaces with implicit relations, Commun. Korean Math. Soc. 23(1) (2008), 111-124. MR2380234

[2] S. Chauhan and B. D. Pant, Common fixed point theorem for weakly compatible mappings in Menger space, J. Adv. Res. Pure Math. 3(2) (2011), 107-119. MR2800793

[3] B. S. Choudhury and K. P. Das, A new contraction principle in Menger spaces, Acta Math. Sinica (English Series) 24(8) (2008), 1379-1386. MR2438308 (2009f:54053)

[4] B. S. Choudhury and K. P. Das, A coincidence point result in Menger spaces using a control function, Chaos, Solitons \& Fractals 42(5) (2009), 3058-3063. MR2560014 (2010j:54062)

[5] R. Chugh, Sumitra and M. A. Khan, Common fixed point theorems for converse commuting maps in fuzzy metric spaces, Internat. Math. Forum 6(37) (2011), 1845-1851. 
[6] J.-x. Fang, Common fixed point theorems of compatible and weakly compatible maps in Menger spaces, Nonlinear Anal. 71(5-6) (2009), 1833-1843. MR2524396 (2010g:54045)

[7] J.-x. Fang and Y. Gao, Common fixed point theorems under strict contractive conditions in Menger spaces, Nonlinear Anal. 70(1) (2009), 184-193. MR2468228 (2009k:47164)

[8] M. Imdad, J. Ali and M. Tanveer, Coincidence and common fixed point theorems for nonlinear contractions in Menger PM spaces, Chaos Solitons \& Fractals 42(5) (2009), 3121-3129. MR2562820 (2010j:54064)

[9] G. Jungck, Compatible mappings and common fixed points, Internat. J. Math. Math. Sci. 9(4) (1986), 771-779. MR0870534 (87m:54122)

[10] G. Jungck and B. E. Rhoades, Fixed points for set valued functions without continuity, Indian J. Pure Appl. Math. 29(3) (1998), 227-238. MR1617919

[11] S. Kumar and B. Fisher, A common fixed point theorem in fuzzy metric space using property (E.A.) and implicit relation, Thai J. Math. 8(3) (2010), 439-446. MR2763666 (2011m:54045)

[12] S. Kumar and B. D. Pant, Common fixed point theorems in probabilistic metric spaces using implicit relation and property (E.A), Bull. Allahabad Math. Soc. 25(2) (2010), 223-235. MR2779240

[13] Z. Lü, Common fixed points for converse commuting selfmaps on a metric space, Acta Anal. Funct. Appl. (Chinese) 4(3) (2002), 226-228. MR1956719

[14] Q.-k. Liu and X.-q. Hu, Some new common fixed point theorems for converse commuting multi-valued mappings in symmetric spaces with applications, Nonlinear Anal. Forum 10(1) (2005), 97-104. MR2162343 (2006d:47102)

[15] K. Menger, Statistical metrics, Proc. Nat. Acad. Sci. U.S.A. 28 (1942), 535-537.

[16] D. Mihet, A note on a common fixed point theorem in probabilistic metric spaces, Acta Math. Hungar. 125(1-2) (2009), 127-130. MR2564425

[17] S. N. Mishra, Common fixed points of compatible mappings in PM-spaces, Math. Japonica 36(2) (1991), 283-289. MR1095742

[18] B. D. Pant and S. Chauhan, A contraction theorem in Menger space, Tamkang J. Math. 42(1) (2011), 59-68. MR2815806

[19] B. D. Pant and S. Chauhan, Common fixed point theorems for two pairs of weakly compatible mappings in Menger spaces and fuzzy metric spaces, Sci. Stud. Res. Ser. Math. Inform. 21(2) (2011), 81-96. MR2956670

[20] B. D. Pant, S. Chauhan and Q. Alam, Common fixed point theorem in probabilistic metric space, Kragujevac J. Math. 35(3) (2011), 463-470. MR2881141

[21] H. K. Pathak and R. K. Verma, Integral type contractive condition for converse commuting mappings, Internat. J. Math. Anal. (Ruse) 3(24) (2009), 1183-1190. MR2604358

[22] H. K. Pathak and R. K. Verma, An integral type implicit relation for converse commuting maps, Internat. J. Math. Anal. (Ruse) 3(24) (2009), 1191-1198. MR2604359

[23] V. Popa, Some fixed point theorems for compatible mappings satisfying an implicit relation, Demonstratio Math. 32(1) (1999), 157-163. MR1691726

[24] V. Popa and D. Turkoğlu, Some fixed point theorems for hybrid contractions satisfying an implicit relation, Stud. Cercet. Ştinţ. Ser. Math. Univ. Bacău (1998), no. 8, 75-86. MR1993808 
[25] R. Saadati, D. O'Regan, S. M. Vaezpour and J. K. Kim, Generalized distance and common fixed point theorems in Menger probabilistic metric spaces, Bull. Iranian Math. Soc. 35(2) (2009), 97-117. MR2642929

[26] B. Schweizer and A. Sklar, Statistical metric spaces, Pacific J. Math. 10 (1960), 313-334. MR0115153 (22 \#5955)

[27] S. Sharma and B. Deshpande, On compatible mappings satisfying an implicit relation in common fixed point consideration, Tamkang J. Math. 33(3) (2002), 245-252. MR1923113 (2003f:47098)

Sunny Chauhan

Near Nehru Training Centre,

H. No. 274, Nai Basti B-14,

Bijnor-246701, Uttar Pradesh, India.

E-mail: sun.gkv@gmail.com

M. Alamgir Khan

Department of Natural Resources Engineering and Management, University of Kurdistan,

Hewler, Iraq.

E-mail: alam3333@gmail.com

Wutiphol Sintunavarat

Department of Mathematics, Faculty of Science,

King Mongkut's University of Technology Thonburi (KMUTT),

Bangkok 10140, Thailand.

E-mail: poom_teun@hotmail.com 